(C) 2010 IEEE. Personal use of this material is permitted. Permission from IEEE must be obtained for all other uses, in any current or future media, including reprinting/republishing this material for advertising or promotional purposes, creating new collective works, for resale or redistribution to servers or lists, or reuse of any copyrighted component of this work in other works. 


\title{
Hypoglycaemia Detection for Type 1 Diabetic Patients Based on ECG Parameters Using Fuzzy Support Vector Machine
}

\author{
Nuryani, S. H. Ling, Member, IEEE and Hung T. Nguyen, Senior Member, IEEE
}

\begin{abstract}
Nocturnal hypoglycaemia in type 1 diabetic patients can be dangerous in which symptoms may not be apparent while blood glucose level decreases to very low level, and for this reason, an effective detection system for hypoglycaemia is crucial. This research work proposes a detection system for the hypoglycaemia based on the classification of electrocardiographic (ECG) parameters. The classification uses a Fuzzy Support Vector Machine (FSVM) with inputs of heart rate, corrected QT $\left(\mathrm{QT}_{\mathrm{c}}\right)$ interval and corrected $\mathrm{TpTe}\left(\mathrm{TpTe}_{\mathrm{c}}\right)$ interval. Three types of kernel functions (radial basis function (RBF), exponential radial basis function (ERBF) and polynomial function) are investigated in the classification. Moreover, parameters of the kernel functions are tuned to find the optimum of the classification. The results show that the FSVM classification using RBF kernel function demonstrates better performance than using SVM. However, both classifiers result approximately same performance if ERBF and polynomial kernel functions are used.
\end{abstract}

\section{INTRODUCTION}

Hypoglycaemia, or abnormally low blood glucose, is a common complication of insulin-dependent diabetes mellitus (IDDM) and remains a central problem [1]. In a number of studies, hypoglycaemia is one of known causes of death in the diabetic patients [2, 3]. Symptomatic hypoglycaemia is categorized as mild to severe episodes. In another study, mild hypoglycaemia occurs in $58 \%$ and severe hypoglycaemia (defined as the reactions to hospitalization or need of assistance) happens in $26 \%$ of 172 insulin-treated diabetic patients [4].

The Diabetes Control and Complications Trial (DCCT) estimated that around $55 \%$ of severe hypoglycaemia episodes occur during sleep [5]. Nocturnal hypoglycaemia can be dangerous in which symptoms may not be apparent while blood glucose level (BGL) decreases to very low level. It is reported that falling plasma glucose to $2.2 \mathrm{mmol} / \mathrm{l}$ very rarely provoke an awakening response in IDDM patients observed and it corresponds to an absence of clear-cut counterregulatory hormonal responses [6] and therefore a detection system for the onset of hypoglycaemia is crucial.

Regarding correlation between hypoglycaemia and cardiac dysrithmia, a number of studies have demonstrated that hypoglycaemia results altered ventricular repolarization or

Manuscript received January, 2010. Nuryani, S. H. Ling and Hung T. Nguyen are with the Centre for Health Technologies, Faculty of Engineering and Information Technology, University of Technology, Sydney, Broadway, NSW 2007, Australia. e-mail contacts: nnuryani@eng.uts.edu.au; Steve.Ling@uts.edu.au; Hung.Nguyen@uts. edu.au prolonged corrected QT $\left(\mathrm{QT}_{\mathrm{c}}\right)$ interval [7-9]. QT interval represents the duration of ventricular depolarization and subsequent repolarization to occur and, in the heart's electrical cycle, is the interval from the start of the $\mathrm{Q}$ wave to the end of the $\mathrm{T}$ wave. $\mathrm{QT}_{\mathrm{c}}$ prolongation in hypoglycaemia is predicted on account of sympathoadrenal stimulation [8]. Moreover, hypoglycaemia also induce to increase heart rate (heartbeats per unit of time) [10].

Therefore, the electrocardiography (ECG) parameters are examined in experiments for hypoglycaemia detection and have demonstrated as essential inputs for system to determine hypoglycaemia episodes. The heart rate as an ECG feature has been applied as one of inputs for detection of the onset of hypoglycaemia using the fuzzy estimator [11] and the fuzzy neural network [12]. Furthermore, heart rate and $\mathrm{QT}_{\mathrm{c}}$ interval have been implemented for nocturnal hypoglycaemia detection using neural network algorithm [13]. Other ECG parameters: $\mathrm{RR}, \mathrm{RT}_{\mathrm{c}}, \mathrm{T}$ wave amplitude, $\mathrm{T}$ wave skewness and $\mathrm{T}$ wave kurtosis have also been applied to detect onset of hypoglycaemia using artificial neural network (ANN) and linear discriminant analysis (LDA) [14]. $\mathrm{RT}_{\mathrm{c}}$ interval and $\mathrm{T}$ wave amplitude have also investigated as inputs for the Rule Base for the nocturnal hypoglycaemia detection [15]. Another ECG parameter is a corrected TpTe $\left(\mathrm{TpTe}_{\mathrm{c}}\right)$ interval that is the interval from the peak to the end of $\mathrm{T}$ wave and is a descriptor of T wave morphology [16-18].

In general, most of the aforementioned approaches for the hypoglycaemia detection employ methods to reach satisfactory level of reliability so as to act as hypoglycaemia detection system using ECG parameters. Up to now the methods still require extensive validation before they can be adopted for worldwide clinical practices. Thus, this research effort is to develop methods to achieve satisfactory of the hypoglycaemia detection. The construction methods in this research is based on classification techniques using fuzzy support vector machine (FSVM), that is not yet explored widely in the onset of hypoglycaemia detection system.

FSVM is a further classification technique of support vector machine (SVM). SVM has proved mostly good performance for classification in various application [19] including in application to classify features of cardiac signals [20-25]. Choosing SVM as a classification tool consider to its good performance and SVM classification ability to generalize well even with small size sample [26]. In the FSVM method, a fuzzy membership is introduced to each training sample of SVM and therefore different training 
points can make different contributions to the learning of decision surface. For that reason, FSVM technique can enhance traditional SVM in reducing effects of outliers and noises in data points [27, 28]. FSVM has showed better performances than SVM in applications such as classification of EEG signals using wavelet-based features [29], multi-class text categorization [30] and image classification [31].

In this paper, the performances of FSVM and SVM classifiers are compared in the classification of three ECG parameters (heart rate, $\mathrm{QT}_{\mathrm{c}}$ and $\mathrm{TpTe}_{\mathrm{c}}$ ) to hypoglycaemia or normoglycaemia. Kernel functions, are implemented for mapping of the data sets to high dimensional space, are also compared to find the best performance of the classification. The kernel functions are radial basis function (RBF), exponential radial basis function (ERBF) and polynomial function. Therefore this work would contribute to demonstrate the FSVM and SVM classification for these ECG parameters in the detection of hypoglycaemia.

The rest of this paper is organized as follows. Methods consisting of features extraction of the ECG signals and the FSVM and SVM classification are described in section II. Section III presents the experimental results and the conclusion for this research is drawn in Section IV.

\section{METHODS}

This work consists of two main stages, feature extraction and FSVM classification (Fig 1). The main task of the feature extraction is to obtain the heart rate, $\mathrm{QT}_{\mathrm{c}}$ and $\mathrm{TpTe}_{\mathrm{c}}$ from the ECG signals. The FSVM classification is constructed to classify the ECG signal inputs into hypoglycaemic or normoglycaemic condition.



Fig. 1. FSVM classification for the ECG signals to hypoglycaemic or normoglycaemic condition.

\section{A. Feature extraction}

The three ECG parameters are estimated based on the ECG signals acquired from the diabetic patients ECG signals recorded by the Compumedics system. The estimation is created in Matlab ${ }^{\circledR}$ environment. Three interval values (RR interval, QT interval and TpTe interval) are illustrated in Fig. 2. The three intervals are then calculated to find heart rate which equals to $60 / \mathrm{RR}, \mathrm{QT}_{\mathrm{c}}$ which equals to $\mathrm{QT} /(\mathrm{RR})^{1 / 2}$ and $\mathrm{TpTe}_{\mathrm{c}}$ which equals to TpTe/(RR) $)^{1 / 2}$. The end of $\mathrm{T}$ wave
( $\mathrm{T}_{\text {end }}$ ) is defined using the Philips QT Interval Measurement Algorithms, that is by drawing a line segment from the top of the $\mathrm{T}$ wave forward in time to a point and the $\mathrm{T}_{\text {end }}$ is a point that has the maximum vertical distance between the point and the line segment [32]. The three ECG parameters are then used as inputs of the classification.

The inputs are labeled with -1 for hypoglycaemia and are labeled with +1 for normoglycaemia. The threshold for hypoglycaemic level used in this system is $3.3 \mathrm{mmol} / \mathrm{l}$. It means that the ECG conditions with BGL equal or lower than $3.3 \mathrm{mmol} / 1$ are defined as hypoglycaemia and those of which are higher than $3.3 \mathrm{mmol} / \mathrm{l}$ are defined as normoglycaemia.

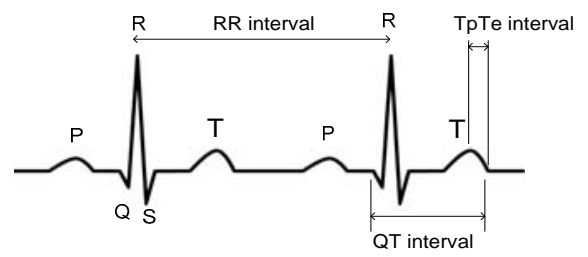

Fig. 2. RR, QT and TpTe intervals in a typical ECG signal

\section{B. Classification using FSVM}

Considering to the three parameters, the classifier is used to automatically recognize hypoglycaemia condition of the type 1 diabetic patients. This section will briefly discuss SVM and FSVM. A more detailed discussion of SVM can be found in [33, 34] and of FSVM in [28, 35].

1) SVM classifier

Fig. 3 shows the optimal hyperplane in the linearly separable binary classification problems. Suppose that there are $k$ training data samples $\left(\mathbf{x}_{i}, y_{i}\right) \ldots\left(\mathbf{x}_{k}, y_{k}\right)$ where $\boldsymbol{x}_{i} \in R^{\mathrm{N}}$ is an- $N$ dimensional space and the associated $y_{i} \in\{+1,-1\}$ is class label. It is assumed that the samples can be separated by the hyperplane satisfying

$$
\mathbf{w} \cdot \mathbf{x}+b=0
$$

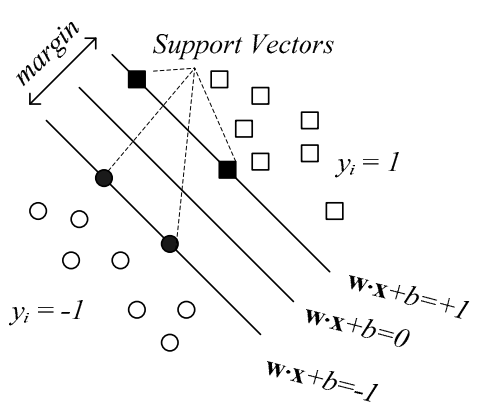

Fig. 3. SVM classification for the linearly sparable case. The lines are the hyperplanes. Squares and circles denotes training data.

where $\mathbf{w}$ is the hyperplane perpendicular vector, $|b| /\left\|\mathbf{w}^{2}\right\|$ is distance of the hyperplane to the origin ( $|b|$ is absolute value of $b$ and $\|\mathbf{w}\|$ is module of $\mathbf{w}$ ). For linearly separable case, SVM algorithm maximize margin between classes and thus all training data satisfy $\mathbf{w} \cdot \mathbf{x}_{i}+b \geq 1$ for $y_{i}=+1$ and $\mathbf{w} \cdot \mathbf{x}_{i}+b \leq 1$ for $y_{i}=-1$. These two constraints can be formulated in one expression, 


$$
y_{i}(\mathbf{w} \cdot \mathbf{x}+b)-1 \geq 0 .
$$

The training data points that satisfy the equality in inequality (2) are called support vectors. The margin between two hyperplanes, $\mathbf{w} \cdot \mathbf{x}+b=+1$ and $\mathbf{w} \cdot \mathbf{x}+b=-1$, is $2 /\|\mathbf{w}\|$. The optimum separating hyperplane can be found by maximizing the margin, minimizing $\left\|\mathbf{w}^{2}\right\|$, with respect to constraint (2).

It is often that in many real world problems that a separating hyperplane does not exist. Hence it is introduced positive slack variable $\xi_{i}$ and then

$$
y_{i}(\mathbf{w} \cdot \mathbf{x}+b) \geq 1-\xi_{i} \text {. }
$$

The optimal separating hyperplane is determined by $\mathbf{w}$ that minimizes the functional

$$
C \sum \xi_{i}+\frac{1}{2}\|\mathbf{w}\|^{2} .
$$

The $C$ is a user defined constant that to control the tradeoff between complexity and proportion of nonseparable points.

Searching the optimal hyperplane in (1) using Lagrange multiplier approach is to maximize

$$
L(\alpha)=\sum_{i=1}^{k} \alpha_{i}-\frac{1}{2} \sum_{i=1}^{k} \sum_{j=1}^{k} \alpha_{i} \alpha_{j} y_{i} y_{j}\left(\mathbf{x}_{i} \cdot \mathbf{x}_{j}\right) .
$$

subject to

$$
0 \leq \alpha_{i} \leq C \text { and } \sum y_{i} \alpha_{i}=0,
$$

where $\alpha_{i}$ is the Lagrange multiplier.

In a case of imbalanced distributions, it is needed to use different error weights and in order to penalize more heavily the undesired type of error, and/or the errors related to the class with the smallest population [36]. Then (4) is modified by minimizes the functional

$$
C^{-} \sum_{i: y_{i}=-1} \xi_{i}+C^{+} \sum_{i: y_{i}=+1} \xi_{i}+\frac{1}{2}\|\mathbf{w}\|^{2}
$$

Having determined the optimum Lagrange multiplier, the optimum solution for the vector $\mathrm{w}$ is given by

$$
\mathbf{w}=\sum \alpha_{i} y_{i} \mathbf{x}_{i}
$$

Replacing the inner-product in (5) with a kernel function $K\left(\mathbf{x}_{\mathbf{i}}, \mathbf{x}_{\mathrm{j}}\right)$ map input data to higher dimensional space so that nonlinearly separable data can be linearly classified. In this paper the classification apply three kernel functions: RBF, ERBF and polynomial functions. Parameters $\sigma$ in the $\mathrm{RBF} / \mathrm{ERBF}$ and $d$ in the polynomial are tuned to obtain a high classification performance. The kernel functions are:

$$
\begin{aligned}
& \text { RBF, } \quad K\left(\mathbf{x}_{i}, \mathbf{x}_{j}\right)=\exp \left(-\frac{\left\|\mathbf{x}_{i}-\mathbf{x}_{j}\right\|^{2}}{2 \sigma^{2}}\right) ; \\
& \text { ERBF, } \quad K\left(\mathbf{x}_{i}, \mathbf{x}_{j}\right)=\exp \left(-\frac{\left\|\mathbf{x}_{i}-\mathbf{x}_{j}\right\|}{2 \sigma^{2}}\right) ;
\end{aligned}
$$

and polynomial, $K\left(\mathbf{x}_{i}, \mathbf{x}_{j}\right)=\left(\mathbf{x}_{i} \cdot \mathbf{x}_{j}+1\right)^{d}$

Finally, for any test vector $\mathbf{x} \in R^{N}$, the output of the classification is then given by

$$
y=\operatorname{sgn}\left(\sum \alpha_{i} y_{i} K\left(\mathbf{x}_{i}, \mathbf{x}\right)\right) .
$$

\section{2) FSVM classifier}

In order to enhance the training performances, fuzzy membership is introduced to each training sample. FSVM introduce a fuzzy membership $0<s_{i}<1$ associated with each data point $\mathrm{x}_{i}$. The output of fuzzy membership $s_{i}$ is regarded as attitude of the corresponding training points toward one class in the classification problem. The optimal hyperplane problem then can be regarded as the solution to [28]

$$
\begin{aligned}
& \text { minimize } C \sum_{i: 1}^{k} s_{i} \xi_{i}+\frac{1}{2} \mathbf{w} \cdot \mathbf{w} \\
& \text { subject to (3). }
\end{aligned}
$$

The problem (10) can be transformed into,

$$
\sum_{i=1}^{k} \alpha_{i}-\frac{1}{2} \sum_{i=1}^{k} \sum_{j=1}^{k} \alpha_{i} \alpha_{j} y_{i} y_{j}\left(\mathbf{x}_{i} \cdot \mathbf{x}_{j}\right)
$$

subject to

$$
0 \leq \alpha_{i} \leq s_{i} C \text { and } \sum y_{i} \alpha_{i}=0 .
$$

In this paper, the output value of the fuzzy membership $s_{i}$ is designed as shown in the Fig 4. This membership is based on the associated blood glucose level (BGL) value $\beta$ of the ECG signals. The peak of $s_{i}$ is at $\beta=3.30 \mathrm{mmol} / \mathrm{l}$ that is the boundary between hypoglycaemia and normoglycaemia. The values of 2.2201 and 6.2201 are considered to the minimum and maximum of BGL data, respectively, in this work. The both values are determined considering to the minimum and maximum value of the BGL data. The output value $s_{i}$ of the membership function is described below:

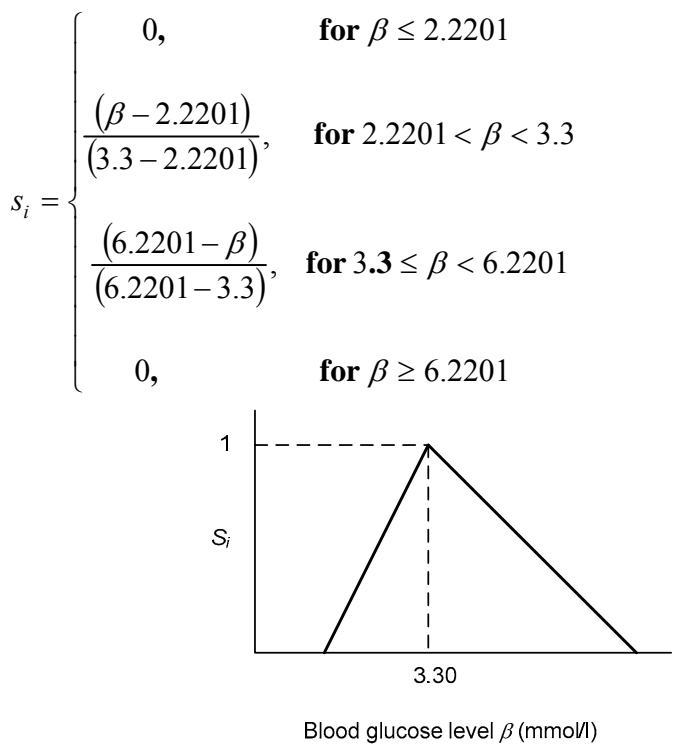

Fig. 4. The fuzzy membership of the FSVM 


\section{EXPERIMENTAL RESULT}

The profile of the blood glucose level (BGL) of the type-1 diabetic patients acquired using the Yellow Spring Analyzer is presented in Fig. 5. The glucose level profile is started with the normal level and then the hypoglycaemic periods occur until the glucose level is less than $3.3 \mathrm{mmol} / \mathrm{l}$. The ECG data of the five type- 1 diabetic patients, with age of $16 \pm 0.7$ years, are investigated for the classification in this work. Each patient data is an overnight monitoring for the natural occurrence of nocturnal hypoglycaemia. In general, the BGL was estimated every 5 minutes and the ECG signals is recorded and stored in ProFusion software (Compumedics).

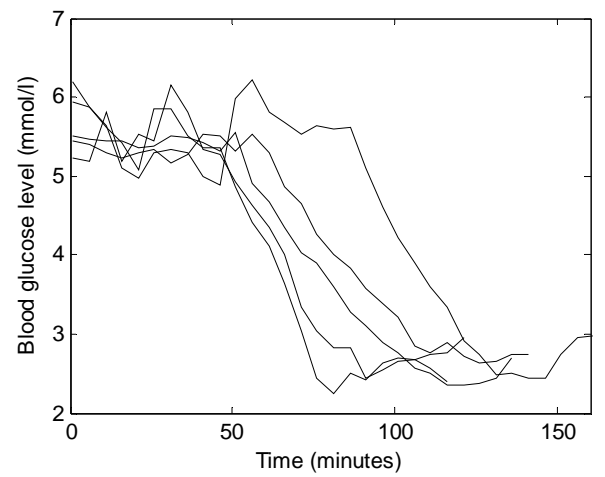

Fig. 5. Blood glucose level profile of the 5 patients

The inputs for the classifications have been obtained from the feature extraction of the ECG signals of the five patients. The data points of the input set are 134 containing both hypoglycaemic and normoglycaemic conditions. The inputs have also been classified using SVM and FSVM classification considering to the hypoglycaemic conditions.

A leave-one-out cross-validation scheme is used to evaluate the performance of the classification. In this scheme, the dataset is divided into 5 subsets with one used for testing and the remaining subsets used to train and construct the SVM decision surface. The performances are measured in terms of sensitivity, specificity and accuracy:

$$
\begin{aligned}
& \text { Sensitivity }=\frac{T P}{T P+F N}, \\
& \text { Specificity }=\frac{T N}{T N+F P}, \\
& \text { Accuracy }=\frac{T P+T N}{T P+T N+F P+F N},
\end{aligned}
$$

where TP (true positive) is number of the inputs that correspond to hypoglycaemia classified as hypoglycaemia. $F P$ (false positive) is number of the inputs that correspond to normoglycaemia classified as hypoglycaemia. TN (true negative) is number the inputs that correspond to normoglycaemia classified as normoglycaemia. $F N$ (false negative) is the inputs that correspond to hypoglycaemia classified as normoglycaemia. Average of sensitivity, specificity, and accuracy of the cross-validation are used for comparison among the classification techniques,

Each of the three kernel functions (RBF, ERBF and polynomial function) has been applied in both SVM and
FSVM classification and the result of the cross-validation is described in Fig. 6 and Fig. 7. The classifiers apply the three kernels with variation of kernel parameters: $\sigma$ parameter (kernel width) is for RBF and ERBF kernel function and $d$ parameter (degree of polynomial) is for polynomial kernel function. Parameter of $\sigma$ is varied from 1 to 141 with step of 10 and parameter of $d$ is varied from 1 to 10 with step of 1 . Considering to the experiments, these steps are sufficient in which the performances of the classifiers with these steps can represent the performances along these kernel parameter ranges.

The graphs show that the choosing kernel functions in the both SVM and FSVM classifiers is crucial to find a good performance in the classification, especially in term of sensitivity for this case. In this experiment, in general, the classifiers with RBF kernel function outperform than the others. Furthermore, tuning the kernel parameters is also significant in the both FSVM and SVM classification. In the classification with RBF kernel width from 1 to 141 , the sensitivity increase but the specificity decrease. Meanwhile, the sensitivity and specificity remain constant during variation of the ERBF kernel width. Using RBF kernel function, the FSVM generally outperform the SVM. Although in the RBF kernel width $\sigma=131$ the sensitivity of both FSVM and SVM is nearly same, the specificity and accuracy of the FSVM is higher. However, both classifiers obtain almost same performance when they use ERBF kernel function. Therefore, choosing how to map the data sets to high dimensional space in SVM and FSVM is crucial.

In the cross-validation, the comparison of the optimum values of the classifications is showed in Table 1. Considering to the classification results having the highest sensitivities, the FSVM classifier with RBF kernel function outperform the SVM classifier that it is indicated by the significantly higher specificity and accuracy that is $58.54 \%$ and $63.20 \%$ respectively. However, using ERBF and polynomial kernel functions, the optimum values of both classifiers are nearly same.

The better performances of the classification using FSVM compared to SVM are in line with the fact that there are more choices for appropriate parameters in the FSVM training than in the SVM training $[28,35]$. The additional parameter is the fuzzy membership that can control the trade-off of the respective training data point. The fuzzy membership is made in the range from close to zero to 1 that consider to the associated BGL values of the ECG parameters (Fig. 4). The lower membership values for the very low and very high BGL values are regarded to the fact that not the all patients have very low or very high BGL. On the other word, the very low or very high value can not represent well of a class in the training and hence it is given the lower membership. For the future, an optimization system could be applied to determine the best of these values to find the better performance of the classification 




(a) The SVM classification with RBF kernel function

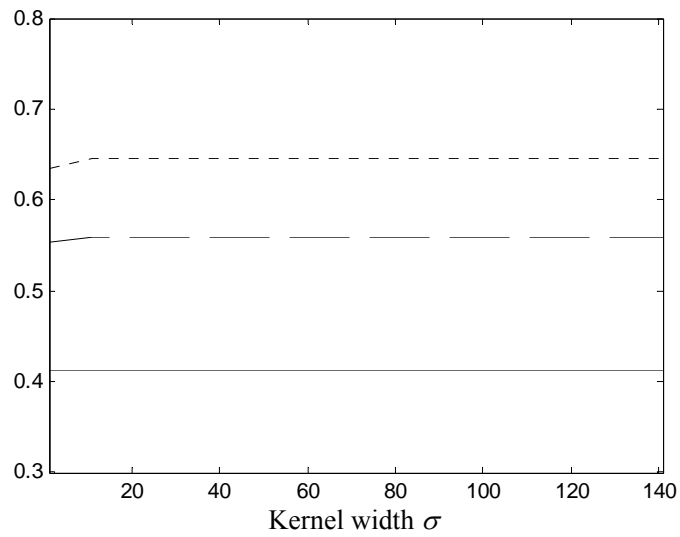

(b) The SVM classification with ERBF kernel function

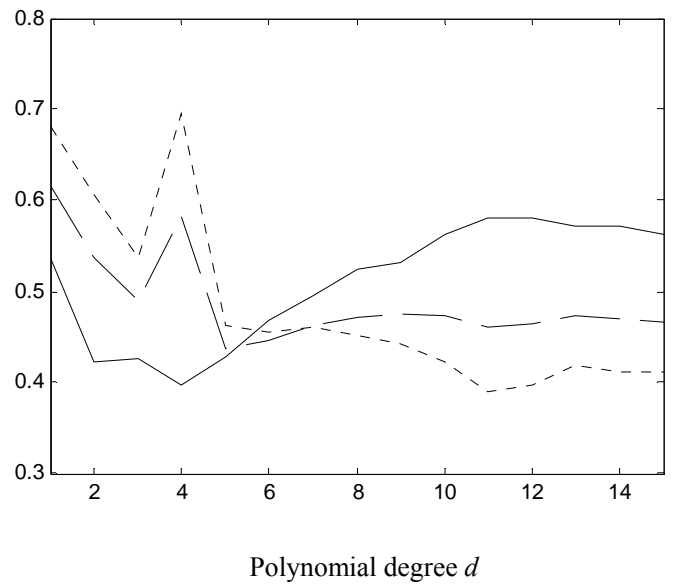

(c) The SVM classification with polynomial kernel tunction

Fig. 6. Cross-validation results of the classifications using the SVM with different kernels and kernel parameters. (Solid line: sensitivity, dotted line: specificity, dashed line: accuracy).

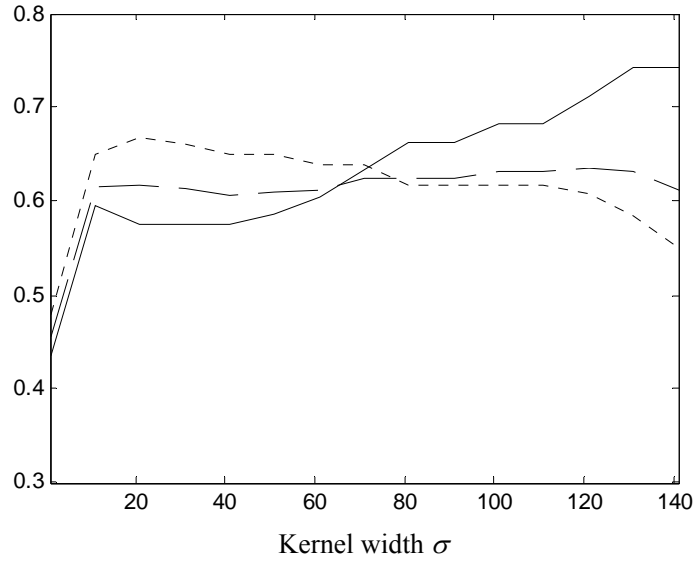

(a) The FSVM classification with RBF kernel function

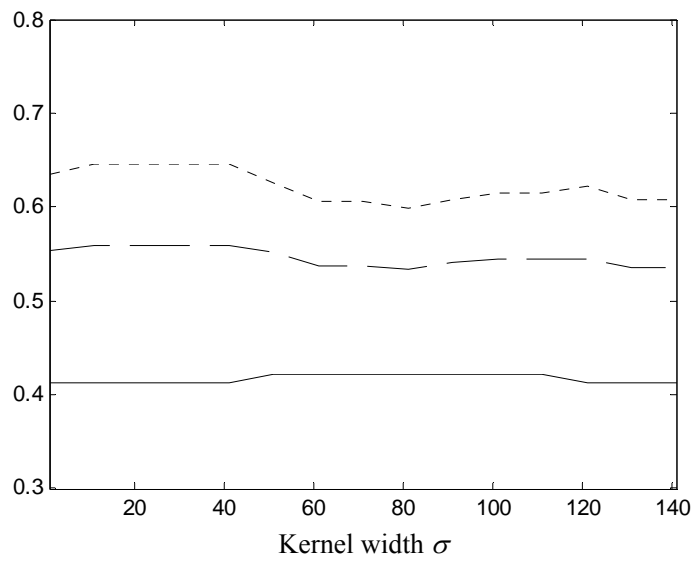

(b) The FSVM classification with ERBF kernel function

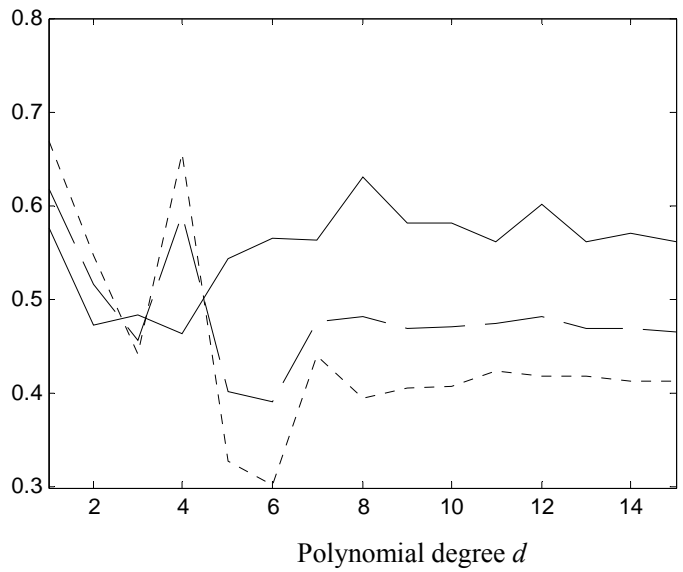

(c) The FSVM classification witn poiynomıaı kerneı runction

Fig. 7. Cross-validation results of the classifications using the FSVM with different kernels and kernel parameters. (Solid line: sensitivity, dotted line: specificity, dashed line: accuracy). 
TABLE 1

OPTIMUM VALUE OF THE LEAVE-ONE-OUT CLASSIFICATION PERFORMANCE OF THE SVM AND FSVM

\begin{tabular}{|c|c|c|c|c|}
\hline Classifier & $\begin{array}{c}\text { Kernel } \\
\text { Function }\end{array}$ & $\begin{array}{c}\text { Sens } \\
\text { (\%) }\end{array}$ & $\begin{array}{c}\text { Spec } \\
\text { (\%) }\end{array}$ & $\begin{array}{c}\text { Acc } \\
\text { (\%) }\end{array}$ \\
\hline \multirow{4}{*}{ SVM } & $\boldsymbol{R B F}$ & 74.43 & 50.50 & 57.34 \\
\cline { 2 - 5 } & ERBF & 41.24 & 64.50 & 55.99 \\
\cline { 2 - 5 } & Polynomial & 57.14 & 41.24 & 46.98 \\
\hline \multirow{6}{*}{ FSVM } & $\boldsymbol{R B F}$ & $\underline{74.19}$ & $\underline{58.54}$ & $\underline{63.20}$ \\
\cline { 2 - 5 } & ERBF & 42.19 & 62.54 & 5510 \\
\cline { 2 - 5 } & Polynomial & 63.05 & 39.60 & 48.25 \\
\hline
\end{tabular}

Sens: Sensitivity; Spec: Specificity; Acc: Accuracy

\section{CONCLUSION}

In this paper, the classifications for the ECG parameters using FSVM and SVM to obtain the hypoglycaemia episodes of the type 1 diabetic patients have been developed. The performances in the cross-validation of both classifiers have also been compared. In this classification, using RBF kernel function and with kernel width of 131, the FSVM demonstrate higher performance of classification than SVM. However, both classifiers applying ERBF and polynomial kernel functions have nearly same performances. In short, the FSVM with RBF kernel function gives $74.14 \%$ sensitivity and $58.54 \%$ specificity for type 1 diabetic problem.

\section{REFERENCES}

[1] T. W. Jones and E.A. Davis, "Hypoglycemia in children with type 1 diabetes: current issues and controversies." Pediatric Diabetes, vol. 4, 2003, pp. 143-150.

[2] F. A. Connell and J. M. Louden, "Diabetes mortality in persons under 45 years of age," American Journal of Public Health, vol. 73, pp. 1174$1177,1983$.

[3] W. M. G. Tunbridge, "Occasional Survey. Factors contributing to deaths of diabetics under fifty years of age. On behalf of the Medical Services Study Group and British Diabetic Association.," The Lancet, vol. 318, pp. 569-572, 1981

[4] C. Goldgewicht, G. Slama, L. Papoz, and G. Tchobroutsky, "Hypoglycaemic reactions in 172 Type 1 (insulin-dependent) diabetic patients," Diabetologia, vol. 24, pp. 95-99, 1983.

[5] The-DCCT-Research-Group, "Epidemiology of severe hypoglycemia in the diabetes control and complications trial," The American Journal of Medicine, vol. 90, pp. 450-459, 1991.

[6] B. Schultes, K. Jauch-Chara, S. Gais, M. Hallschmid, E. Reiprich, W. Kern, K. M. Oltmanns, A. Peters, H. L. Fehm, and J. Born, "Defective awakening response to nocturnal hypoglycemia in patients with type 1 diabetes mellitus," PLoS Med, vol. 4, p. e69, 2007.

[7] J. L. B. Marques, E. George, S. R. Peacey, N. D. Harris, I. A. Macdonald, T. Cochrane, and S. R. Heller, "Altered ventricular repolarization during hypoglycaemia in patients with diabetes," Diabetic Medicine, vol. 14, pp. 648-654, 1997.

[8] R. T. C. E. Robinson, N. D. Harris, R. H. Ireland, S. Lee, C. Newman, and S. R. Heller, "Mechanisms of abnormal cardiac repolarization during insulin-induced hypoglycemia," Diabetes, vol. 52, pp. 14691474, June 2003

[9] N. P. Murphy, M. E. Ford-Adams, K. K. Ong, N. D. Harris, S. M. Keane, C. Davies, R. H. Ireland, I. A. MacDonald, E. J. Knight, J. A. Edge, S. R. Heller, and D. B. Dunger, "Prolonged cardiac repolarisation during spontaneous nocturnal hypoglycaemia in children and adolescents with type 1 diabetes," Diabetologia, vol. 47, pp. 1940-1947, 2004.

[10] G. Heger, K. Howorka, H. Thoma, G. Tribl, and J. Zeitlhofer,
"Monitoring set-up for selection of parameters for detection of hypoglycaemia in diabetic patients," Medical and Biological Engineering and Computing, vol. 34, pp. 69-75, 1996.

[11] G. Hastings, N. Ghevondian, and H. T. Nguyen, "A self-organising fuzzy estimator for hypoglycaemia monitoring in diabetic patients," in Proceeding of the 20th Annual International Conference of the IEEE Engineering in Medicine and Biology Society, 1998, pp. 1371-1374 vol.3.

[12] N. Ghevondian, H. T. Nguyen, and S. Colagiuri, "A novel fuzzy neural network estimator for predicting hypoglycaemia in insulin-induced subjects," in Proceeding of the 23rd Annual International Conference of the IEEE Engineering in Medicine and Biology Society, 2001, pp. 1657. 1660 vol.2.

[13] H. T. Nguyen, N. Ghevondian, and T. W. Jones, "Detection of nocturnal hypoglycemic episodes (natural occurrence) in children with Type 1 diabetes using an optimal Bayesian neural network algorithm," in Proceeding of the 30th Annual International Conference of the IEEE Engineering in Medicine and Biology Society, 2008, pp. 1311-1314.

[14] C. Alexakis, H. O. Nyongesa, R. Saatchi, N. D. Harris, C. Davies, C. Emery, R. H. Ireland, and S. R. Heller, "Feature extraction and classification of electrocardiogram (ECG) signals related to hypoglycaemia," in Computers in Cardiology, 2003, 2003, pp. 537-540.

[15] C. Alexakis, M. Rodrigues, R. Saatchi, H. O. Nyongesa, C. Davies, R. H. Ireland, N. D. Harris, and S. R. Heller, "A knowledge-based electrocardiogram-monitoring system for detection of the onset of nocturnal hypoglycaemia in type 1 diabetic patients," in Computers in Cardiology, 2006, 2006, pp. 5-8.

[16] P. A. Mads, Q. X. Joel, G. Claus, K. K. JÃ,rgen, T. Egon, and J. S. Johannes, "New descriptors of T-wave morphology are independent of heart rate," Journal of electrocardiology, vol. 41, pp. 557-561, 2008.

[17] K. K. Jorgen, H. Christian, V.-L. Esben, P. A. Mads, G. Claus, J. S. Johannes, T. Poul Erik Bloch, C. Michael, K. J. Henrik, and T. Egon, "TpeakTend interval in long QT syndrome," Journal of electrocardiology, vol. 41, pp. 603-608, 2008.

[18] A. K. Jan, J. R. v. E. Henk, and H. Gerard van, "The meaning of the TpTe interval and its diagnostic value," Journal of electrocardiology, vol. 41, pp. 575-580, 2008.

[19] D. Meyer, F. Leisch, and K. Hornik, "The support vector machine under test," Neurocomputing, vol. 55, pp. 169-186, 2003.

[20] A. Kampouraki, G. Manis, and C. Nikou, "Heartbeat time series classification with support vector machines," IEEE Transactions on Information Technology in Biomedicine, vol. 13, pp. 512-518, 2009.

[21] A. H. Khandoker, M. Palaniswami, and C. K. Karmakar, "Support vector machines for automated recognition of obstructive sleep apnea syndrome from ECG recordings," IEEE Transactions on Information Technology in Biomedicine, vol. 13, pp. 37-48, 2009.

[22] G. Georgoulas, D. Stylios, and P. Groumpos, "Predicting the risk of metabolic acidosis for newborns based on fetal heart rate signal classification using support vector machines," IEEE Transactions on Biomedical Engineering, vol. 53, pp. 875-884, 2006.

[23] S. Osowski, L. T. Hoai, and T. Markiewicz, "Support vector machinebased expert system for reliable heartbeat recognition," IEEE Transactions on Biomedical Engineering, vol. 51, pp. 582-589, 2004.

[24] N. Acir, "A support vector machine classifier algorithm based on a perturbation method and its application to ECG beat recognition systems," Expert Systems with Applications, vol. 31, pp. 150-158, 2006.

[25] S. S. Mehta and N. S. Lingayat, "Application of support vector machine for the detection of P- and T-waves in 12-lead electrocardiogram," Computer Methods and Programs in Biomedicine, vol. 93, pp. 46-60, 2009.

[26] R. P. W. Duin, "Classifiers in almost empty spaces," in Proceeding of the 15th International Conference on Pattern Recognition, 2000. on, 2000, pp. 1-7 vol.2.

[27] C.F. Lin and S.D. Wang, "Training algorithms for fuzzy support vector machines with noisy data," Pattern Recognition Letters, vol. 25, pp. 1647-1656, 2004.

[28] C.F. Lin and S. D. Wang, "Fuzzy support vector machines," IEEE Transactions on Neural Networks, , vol. 13, pp. 464-471, 2002.

[29] Q. Xu, H. Zhou, Y. Wang, and J. Huang, "Fuzzy support vector machine for classification of EEG signals using wavelet-based features," Medical Engineering \& Physics, vol. 31, pp. 858-865, 2009 
[30] T.-Y. Wang and H.-M. Chiang, "Fuzzy support vector machine for multi-class text categorization," Information Processing and Management, vol. 43, pp. 914-929, 2007.

[31] J. Li, S. Huang, R. He, and K. Qian, "Image classification based on Fuzzy Support Vector Machine," in International Symposium on Computational Intelligence and Design, Hubei China, 2008, pp. 68-71.

[32] H. Z. Sophia, D. H. Eric, M. L. James, E. G. Richard, and Q. F. Dirk, "Philips QT interval measurement algorithms for diagnostic, ambulatory, and patient monitoring ECG Applications," Annals of Noninvasive Electrocardiology, vol. 14, pp. S3-S8, 2009.

[33] C. J. C. Burges, "A Tutorial on support vector machines for pattern recognition," Data Mining and Knowledge Discovery, vol. 2, pp. 121$167,1998$.

[34] J. G. S. d. Tejada and J. S. Martinez-Echevarria, "Support vector machines," in Computational Intelligence for Engineering and Manufacturing Springer, 2007, pp. 147-191.

[35] C.F. Lin and S.D. Wang, "Fuzzy support vector machines with automatic membership setting," in Support Vector Machines: Theory and Applications: Springer, 2005, pp. 233-254.

[36] K. Veropoulos, C. Campbell, and N. Cristianini, "Controlling the Sensitivity of Support Vector Machines," in The Sixteenth International Joint Conference on Artificial Intelligence (IJCAI99) Stockholm, Sweden, 1999. 